\title{
Fault Behavior of Power Distribution Networks with Distributed Generation and Uncertainties
}

\author{
Laiz Souto \\ Intelligent Systems and Control Engineering \\ Universitat de Girona \\ 17003 Girona, Girona, Spain \\ laiz.souto@gmail.com
}

\begin{abstract}
This paper assesses the fault behavior of power distribution networks with distributed energy resources and uncertainties related with the representation of different grid components and parameters. The algorithm is based upon an impedance representation of the grid, relying on information about the network topology and electrical parameters of the feeders. In addition, distinct types of loads and distributed energy resources are represented as non-deterministic parameters, as well as the fault impedance and fault distance parameters and errors in the phasor quantities. As an outcome, the implementation of the proposed method shall provide a comprehensive, but accurate estimation of the points of fault by considering a range of possible fault scenarios. Furthermore, the methodology is demonstrated in a small overhead distribution network simulated under different operating conditions. For a given short-circuit current, the results indicate a range of possible fault distances and vice versa.
\end{abstract}

Index Terms-distributed energy resources, fault currents, fault location, modeling, power distribution faults, uncertainty, ZIP loads

\section{INTRODUCTION}

Despite the modernization of electricity infrastructures, accurate fault location in power distribution networks is still a challenging task. The topology of power distribution networks is typically radial, consisting of a primary feeder connected to lateral branches, with fault locators usually centralized at the substation bus. As a consequence, the presence of multiterminal lines and meshes, the non-homogeneity of lines, type of neutral grounding, and inaccurate representation of distributed generation, loads, and fault currents still represent stumbling blocks to accurate fault location in power distribution networks [1].

The impact of errors and uncertainties in fault location has been studied in [2], which obtained the probability functions of variables involved in power system faults from real measurements acquired by power quality monitors in distribution substations; in [3], which evaluated application requirements and modeling limitations and uncertainties to define appropriate fault location methods; in [4], which used pre-fault voltages

This research was supported by the European Union's Horizon 2020 research and innovation programme, call LCE-01-2016-2017, under the auspices of the project "Renewable penetration levered by Efficient Low Voltage Distribution grids", grant agreement number 773715, and University of Girona scholarship. measured by smart meters along the feeders with and without measurement noise in a L1-norm minimization method to test the system performance under distinct fault conditions; in [5], which presented an impedance-based method for fault location considering different sources of errors to evaluate accuracy and robustness; and in [6], which evaluated the sources of errors affecting the accuracy of fault location. In turn, [7][9] evaluate the short-circuit current contribution of distributed energy resources and their impact on power system protection schemes, among others.

Although previous fault location methods have taken into account errors in the measurements and uncertainties in the modeling of loads, distributed generation, and fault impedance parameters, such errors and uncertainties have only been applied deterministically to assess the accuracy and robustness of the proposed algorithms in different scenarios. On the other hand, a thorough evaluation of the effect of such errors and uncertainties on the range of possible results can provide comprehensive information about possible fault scenarios, but is still missing in the previous fault location strategies.

In this context, this article presents a new approach to analyze the fault behavior of power distribution networks, using information about the network topology and electrical parameters of the feeders, stored in the utility's database, together with estimations of loads, distributed generation units, and fault parameters. The text is structured as follows: Section II describes the methodology, Section III features a case study, Section IV presents the results, and Section V summarizes the conclusions.

\section{FAULT ANALYSIS}

This section is divided in three parts: initial assumptions are described in II-A, the short-circuit calculations are explained in II-B, and the modeling of uncertain parameters is finally described in II-C.

\section{A. Assumptions}

This analysis is valid for any power transmission and distribution systems, but especially convenient for power distribution networks with variable loads and distributed energy resources along their feeders. It requests information about the network topology and electrical parameters of the 
feeders, stored in the utility's database, estimations of the operating status of loads, distributed energy resources, and fault impedance parameters, and uncertainties associated with the voltage and current phasor quantities measured at the substation bus. It is based on phase components to deal with unbalanced networks, which implies that all phasor quantities and admittance matrices are expressed in terms of circuit phases. Essentially, the algorithm computes the equivalent admittance matrix of the network, then calculates the fault current at each line section under investigation to determine the fault behavior of the network over a range of scenarios. This procedure is described in the next paragraphs.

\section{B. Algorithm}

The methodology consists of an optimization via simulations approach conducted in OpenDSS. Thereby, the algorithm implemented in this software is briefly introduced in the following paragraphs.

First, the algorithm computes the nodal admittance matrix of the network, denoted by $\bar{Y}$, representing a specific operating condition of the network previously defined in the input data. This procedure includes all loads as admittances, as well as the equivalent admittance of all generation buses. Thus, all generation buses (i.e. slack bus and connection points of distributed generation units) are converted to their Thevenin equivalents. Then, the equivalent system is described by (1), where $\dot{I}$ denotes the vector of current injections into the network and $\dot{V}$ is the vector of node voltage to the ground.

$$
\dot{I}=\bar{Y} \dot{V}
$$

Next, a direct solution of $\bar{Y}$ is calculated with source injections and generator injections. This procedure allows for computation of the resulting open-circuit voltage vector $\dot{V}_{O C}$ and short-circuit admittance matrix $\bar{Y}_{S C}$, from which the shortcircuit current vector $\dot{I}_{S C}$ can be calculated. The short-circuit currents are then computed at each bus using the Thevenin equivalent model as in (2).

$$
\dot{I}_{S C}=\bar{Y}_{S C} \dot{V}_{O C}
$$

The short-circuit currents and fault distances representing a specific operating condition of the network are then saved. The algorithm proceeds with an update in the estimations of uncertain parameters, described as follows in Section II-C, until all operating scenarios of interest are evaluated. A flowchart of the proposed algorithm is schematized in Fig. 1.

\section{Estimations and uncertainties}

The algorithm described in Section II-B may be computed over a range of scenarios representing variations of the fault operating conditions of the network. Thus, consider that the exact operating status of loads, distributed energy resources, and fault impedance parameters are unknown, but can be estimated over a range of values. In addition, assume that the

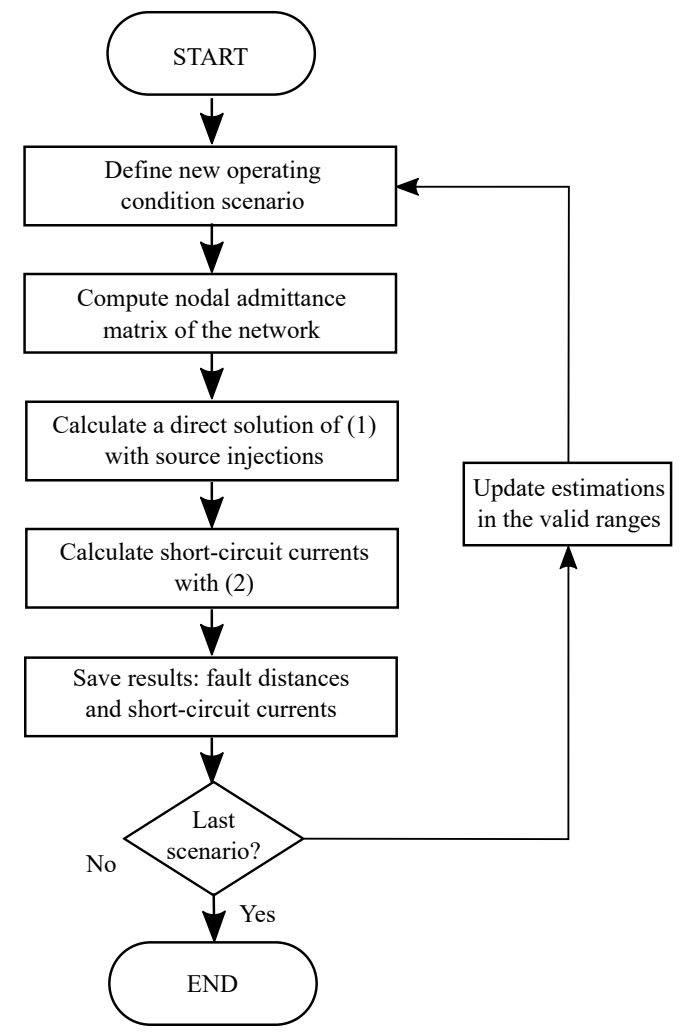

Fig. 1. Flux diagram of the algorithm

phasor quantities may present inaccuracies caused by measurement errors and/or noise. Such uncertainties are further described in the following paragraphs.

The loads of the network may be a combination of constantimpedance, constant-current, and constant-power models. Thereby, the load estimation depends on its corresponding electrical behavior, represented by (3) for a constantimpedance model, by (4) for a constant-current model, and by (5) for a constant-power model.

$$
\begin{gathered}
\bar{Y}_{L}=\frac{\dot{I}_{L}}{\dot{V}_{L}}=\text { constant } \longrightarrow\left\{\begin{array}{l}
\bar{Y}_{L}=\text { constant } \\
\dot{I}_{L} \propto \dot{V}_{L}
\end{array}\right. \\
\dot{I}_{L}=\bar{Y}_{L} \dot{V}_{L}=\text { constant } \longrightarrow\left\{\begin{array}{l}
\bar{Y}_{L} \propto \dot{V}_{L}^{-1} \\
\dot{I}_{L}=\text { constant }
\end{array}\right. \\
\bar{S}_{\text {load }}=\bar{Y}_{L}\left|\dot{V}_{L}\right|^{2}=\text { constant } \longrightarrow\left\{\begin{array}{l}
\bar{Y}_{L} \propto \dot{V}_{L}^{-2} \\
\dot{I}_{L} \propto \dot{V}_{L}^{-1}
\end{array}\right.
\end{gathered}
$$

In (3), $\bar{Y}_{L}$ is known and constant; in (4), $\dot{I}_{L}$ is known and $\bar{Y}_{L}$ may assume a range of values proportionally to $\dot{V}_{L}^{-1}$; in (5), the apparent power $\bar{S}_{\text {load }}$ is known and $\bar{Y}_{L}$ may assume a range of values proportionally to $\dot{V}_{L}^{-2}$. In turn, $\dot{I}_{L}$ is proportional to $\dot{V}_{L}$ in (3) and proportional to $\dot{V}_{L}^{-1}$ in (5). Considering that $\dot{V}_{L}$ is lower and upper bounded, that is, $\dot{V}_{L} \leq \dot{V}_{L} \leq{\overline{\dot{V}_{L}}}_{\text {, the }}$ minimum and maximum values of $\bar{Y}_{L}$ and $\overline{\dot{I}}_{L}$ in (3) to (5) can be determined. 
The voltage and current phasor quantities at the connection points of distributed generation depend on the type of generator and might be obtained from IEDs or estimated otherwise. For distributed generation units interfaced with power electronics, the output current is assumed to be constant and nearly equivalent to the rated current [10]. Thereby, considering a lower and upper bound for $\dot{I}_{D G}$ and $\dot{V}_{D G}$, $\dot{I}_{D G} \leq \dot{I}_{D G} \leq \overline{\dot{I}_{D G}}$ and $\dot{V}_{D G} \leq \dot{V}_{D G} \leq \overline{\dot{V}_{D G}}$ hold. In particular, for P-V generators, $\dot{V}_{D G}$ is fixed and thereby $\dot{I}_{D G}$ is proportional to the active power $\bar{P}_{D G}$ supplied to the grid, according to (6).

$$
\bar{P}_{D G}=\operatorname{Real}\left\{\dot{V}_{D G} \times \dot{I}_{D G} *\right\} \longrightarrow \bar{P}_{D G} \propto\left|\dot{I}_{D G}\right|
$$

Although the fault admittance is assumed to be infinite in Section II-B, a range of finite pre-specified values can be used to calculate the short-circuit currents at a given point of fault denoted by $d$. Considering a lower bound $\underline{Y}_{F}$ and upper bound $\bar{Y}_{F}, \bar{Y}_{F} \leq \bar{Y}_{F} \leq \overline{\bar{Y}}_{F}$ holds. Alternatively, the same fault impedance $\overline{\bar{Y}}_{F}$ can be used to compute the shortcircuit currents at different locations along the feeders such that $0 \leq d \leq d_{\max }$, where $d_{\max }$ is the length of the feeder.

In addition, consider that the voltage and current phasor quantities at the substation bus, denoted by $\dot{V}_{S}$ and $\dot{I}_{S}$, may be inaccurate due to measurement errors and/or noise and that the uncertainties are denoted by $\Delta \dot{V}_{S}, \Delta \dot{I}_{S}$. Thereby, the actual voltage and current phasor quantities are comprised in the ranges $\dot{V}_{S}-\Delta \dot{V}_{S} \leq \dot{V}_{S} \leq \dot{V}_{S}+\Delta \dot{V}_{S}$ and $\dot{I}_{S}-\Delta \dot{I}_{S} \leq$ $\dot{I}_{S} \leq \dot{I}_{S}+\Delta \dot{I}_{S}$. This produces an offset in the short-circuit currents calculated with (2).

As a result, the short-circuit currents calculated deterministically with (2) change as a function of $\bar{Y}_{L}, \dot{I}_{L}, \dot{V}_{L}, \bar{Y}_{D G}, \dot{I}_{D G}$, $\dot{V}_{D G}, \dot{V}_{S}, \dot{I}_{S}, \bar{Y}_{F}$, and $d$. To this extent, a few possible fault scenarios can be investigated so that a range of short-circuit currents can be determined and associated with a specific point of fault. Additionally, if historical data of fault events are available, the uncertainties aforementioned can be associated with specific probability distribution functions such that the conditional probability of occurrence can be determined for each fault scenario under evaluation.

\section{Simulations}

The methodology was simulated and tested in OpenDSS. Three-phase short-circuit simulations were performed in a modified version of the IEEE 4-bus DY-balanced system, whose topology is illustrated in Fig.2. This distribution system has 5 buses and is composed of a $12.47 / 4.16[\mathrm{kV}]$ distribution substation connecting the primary distribution feeder to two lateral branches, where the distributed generation DG1 and the load L1 are installed. The total length of the primary feeder is $2000[\mathrm{ft}]$, whereas the length of each lateral branch is $250[\mathrm{ft}]$.

The external grid is represented as an infinite bus with 200 [MVA] short-circuit power. The load was modeled as $100 \%$ constant power, $100 \%$ constant impedance, and $100 \%$ constant current in distinct scenarios, with a total consumption

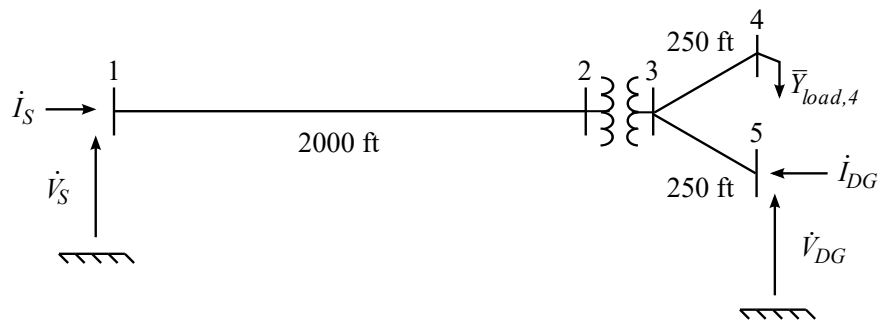

Fig. 2. Tested distribution network: stiff source at bus 1 (slack bus), distribution substation transformer at buses $2-3$, load at bus 4 , and distributed generation at bus 5

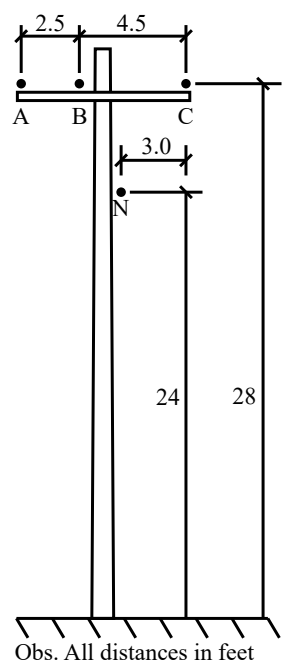

Fig. 3. Arrangement of distribution pole and conductors

between $4.2[\mathrm{MW}]$ and $6.6[\mathrm{MW}]$ (power factor of 0.9). In turn, the distributed generation was modeled as a P-V equivalent source with $\left|\dot{V}_{D G}\right|=1.02[$ p.u.] and injected power varying from $1.0[\mathrm{MW}]$ to $2.0[\mathrm{MW}]$.

The poles and conductors used in the simulation cases, typical of medium voltage distribution level, are displayed in Fig. 3. The line sections are all composed of 336.4 MCM ACSR Linnet phase conductors and 4/0 Penguin ground wires.

The faults were simulated in OpenDSS in fault study mode along the lateral branches where DG1 and L1 are connected, with fault admittances introduced every $25[\mathrm{ft}]$ along these laterals. This provided 21 simulated faults for every possible combination of $\bar{P}_{D G}$ and $\bar{P}_{L}$ (i.e. 10 simulations along each branch and 1 simulation at the connection node per combination of $\left.\bar{P}_{D G}, \bar{P}_{L}\right)$.

The fault scenarios evaluated consist of:

1) $P_{D G}=1.5[\mathrm{MW}]$ fixed and load modeled as $100 \%$ constant power, $100 \%$ constant impedance, and $100 \%$ constant current with $P_{L} \in[4.2,6.6][\mathrm{MW}], 0.6[\mathrm{MW}]$ step;

2) $P_{L}=5.4[\mathrm{MW}]$ fixed in $100 \%$ constant power, $100 \%$ constant impedance, and $100 \%$ constant current models, and distributed generation with $P_{D G} \in[1.0,2.0][\mathrm{MW}]$, $0.25[\mathrm{MW}]$ step;

3) $P_{D G}=1.0[\mathrm{MW}]$ and $P_{L}=6.6[\mathrm{MW}]$ versus $P_{D G}=$ 

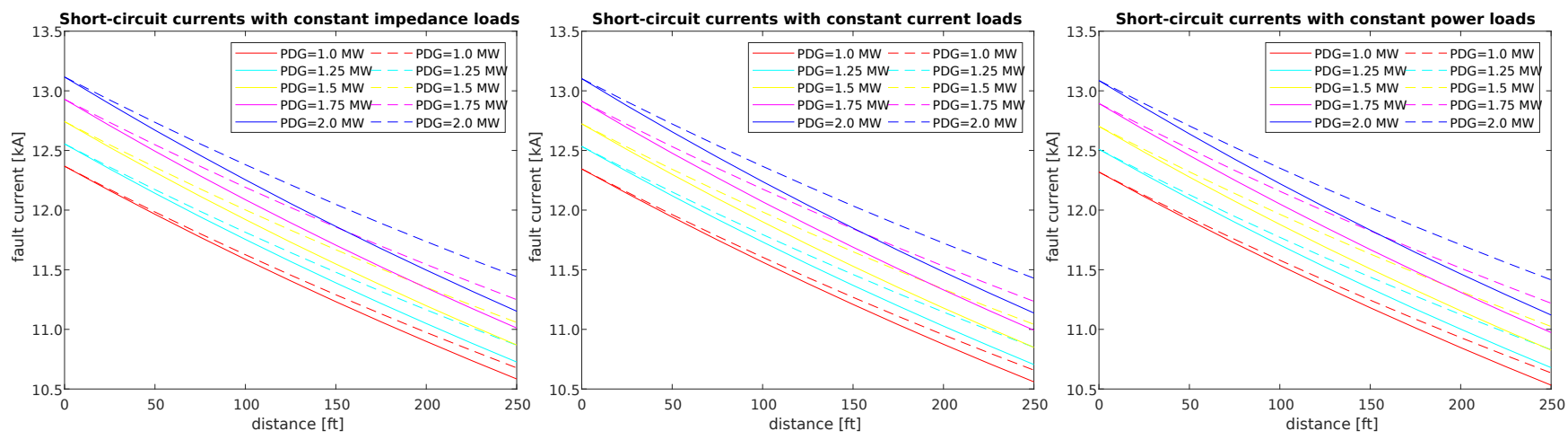

Fig. 4. Short-circuit currents at phase A with varying $P_{D G}$ and $P_{L}=5.4[\mathrm{MW}]$ constant impedance, current, and power load models

$2.0[\mathrm{MW}]$ and $P_{L}=4.2[\mathrm{MW}]$ fixed in $100 \%$ constant power, $100 \%$ constant impedance, and $100 \%$ constant current models.

\section{RESULTS AND DISCUSSION}

This section presents the short-circuit currents obtained over a range of scenarios. For clarity, the results of the case studies aforementioned are illustrated graphically along the lateral branches $3-4$ and $3-5$ of Fig. 2. All graphs of Fig. 4 to 6 show three-phase short-circuit currents simulated at a single phase. Solid lines show the fault currents along the branch $3-4$, whereas dashed lines show the fault currents along the branch $3-5$.

\section{A. Scenario 1}

The results of the proposed method for the first scenario (with varying power consumption and load types) are illustrated graphically in Fig. 4 with a constant impedance, constant current, and constant power load model (graphs of Fig. 4 from the left to the right). A comparison between solid and dashed lines of the same color shows that the differences between the short-circuit currents along the branches $3-4$ and $3-5$ increase as $P_{D G}$ increases. In turn, a comparison between solid and dashed lines of different colors shows that $P_{D G}$ introduces an offset in the short-circuit currents simulated. Moreover, the graphs show that, for the same fault distances, the grid setting with a constant-impedance load presents the highest short-circuit current magnitudes, followed by the constantcurrent load and the constant-power load. However, the shortcircuit levels do not vary significantly with the load model in use. This is explained by the changes made in the equivalent representation of the grid calculated with (2), as the current injections increase as $P_{D G}$ increases.

\section{B. Scenario 2}

The results of the proposed method for the second scenario (varying injected power from distributed generation unit with different load types) are presented in Fig. 5 for a constant impedance, constant current, and constant power load model (graphs of Fig. 5 from the left to the right). A comparison between solid and dashed lines of the same color shows that the differences between the short-circuit currents along the branches $3-4$ and $3-5$ increase slightly as $P_{L}$ decreases. In turn, a comparison between solid and dashed lines of different colors shows that $P_{L}$ introduces a small offset in the short-circuit currents simulated. In addition, the graphs show that, for the same fault distances, the grid setting with a constant-impedance load presents the highest short-circuit current magnitudes, followed by the constant-current load and the constant-power load. However, the short-circuit levels do not vary significantly with the load model in use and remain roughly the same for different values of $P_{L}$. Furthermore, the changes in the fault behavior of the grid presented hereby are much smaller than those verified in Section IV-A with varying $P_{D G}$. This is attributed to the changes made in the Thevenin equivalent calculated with (2); the power injected by the generator changes the current injections of the grid considerably, whereas the load admittance is much smaller than the fault admittance and does not produce significant changes in the equivalent admittance at the point of fault.

\section{Scenario 3}

The results of the proposed method for the third scenario (minimum and maximum fault currents with different load types) are displayed in Fig 6. A comparison between solid and dashed lines of the same color shows that the differences between the short-circuit currents along the branches $3-4$ and $3-5$ increase as $P_{D G}$ increases and $P_{L}$ decreases. In turn, a comparison between solid and dashed lines of different colors shows that the offset in the short-circuit currents decreases with the distance along the branch $3-4$ and increases with the distance along the branch $3-5$.

\section{Interpretation of the results}

The results presented in Sections IV-A to IV-C can be interpreted in two ways: looking at a fixed short-circuit current and looking at a fixed distance. The former shows which points of fault may present the same fault current, whereas the latter shows which fault currents may occur at a given point of fault.

For illustration, consider the simulation results plotted in Fig. 4 in two distinct situations: a $12[\mathrm{kA}]$ fixed short-circuit current and a $200[\mathrm{ft}]$ fixed fault distance. The resulting fault distances and short-circuit currents obtained with different 

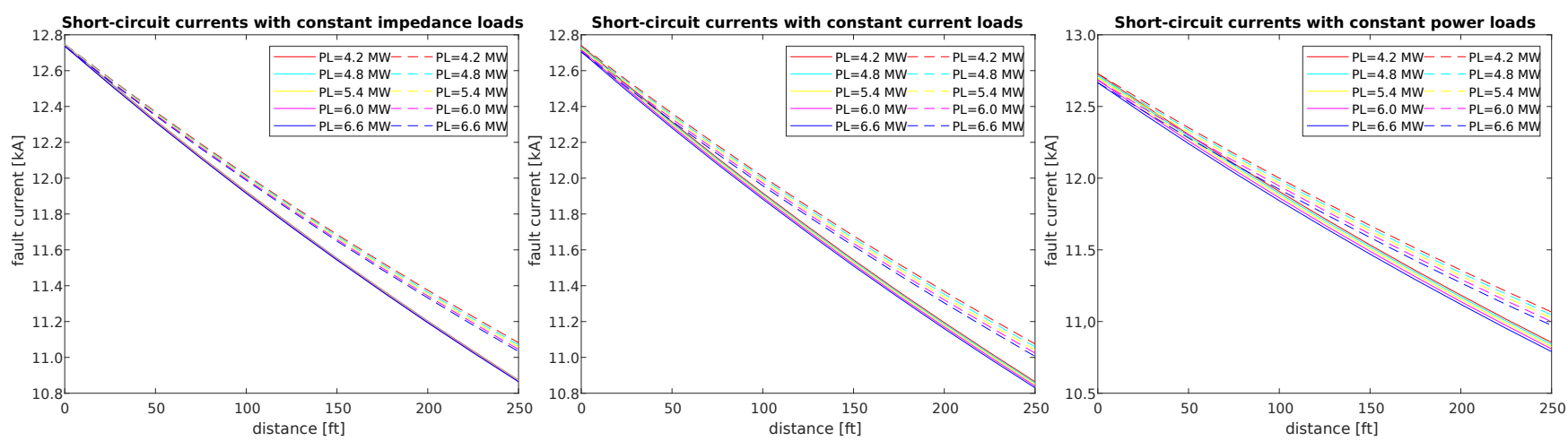

Fig. 5. Short-circuit currents at phase A with varying $P_{L}$ in constant impedance, current, and power load models and $P_{D G}=1.5[\mathrm{MW}]$

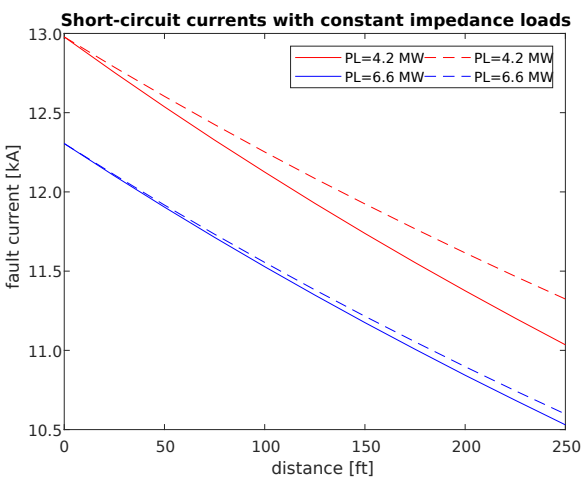

Fig. 6. Minimum and maximum short-circuit currents

operating conditions are summarized in Table I and Table II, respectively.

This analysis shows that a range of possible scenarios has to be evaluated for a fixed short-circuit current and/or fault distance when uncertainties are taken into consideration. Additionally, if historical data of fault events are available, the uncertainties aforementioned can be associated with specific probability distribution functions. For instance, if a shortcircuit is more likely to occur when $P_{L}$ is the highest and $P_{D G}$ is the lowest (blue lines of Fig. 6), then this operating condition shall be prioritized in the search for faults. In this case, for a given short-circuit current, the closest fault distance to node 3 shall be investigated first; for a given point of fault, the smallest short-circuit current shall be considered.

The examples aforementioned may be extended to the graphs of Fig. 5 and Fig. 6 and take into consideration different short-circuit currents and fault distances.

\section{CONCLUSion}

The methodology is able to provide a comprehensive, but accurate estimation of possible fault locations and shortcircuit currents, considering a range of values to estimate loads, distributed generation, and fault distance and impedance parameters, represented as non-deterministic parameters. The results obtained over a range of fault scenarios, representing different operating conditions subject to uncertainties, are comprehensive enough to provide a good overview of the possible fault conditions of the grid. For a fixed short-circuit
TABLE I

RESUlTS: POSSIBLE FAULT DISTANCES CALCULATED WITH A 12 [KA] SHORT-CIRCUIT CURRENT IN FIG. 4

\begin{tabular}{|c|c|c|c|}
\hline Setting & Lateral & $P_{D G}(\mathrm{MW})$ & fault distance $(\mathrm{ft})$ \\
\hline \multirow[t]{10}{*}{ constant-impedance load } & $3-4$ & 1.0 & 46 \\
\hline & & 1.25 & 68 \\
\hline & & 1.5 & 90 \\
\hline & & 1.75 & 111 \\
\hline & & 2.0 & 132 \\
\hline & $3-5$ & 1.0 & 48 \\
\hline & & 1.25 & 74 \\
\hline & & 1.5 & 100 \\
\hline & & 1.75 & 128 \\
\hline & & 2.0 & 157 \\
\hline \multirow[t]{10}{*}{ constant-current load } & $3-4$ & 1.0 & 42 \\
\hline & & 1.25 & 65 \\
\hline & & 1.5 & 87 \\
\hline & & 1.75 & 109 \\
\hline & & 2.0 & 130 \\
\hline & $3-5$ & 1.0 & 45 \\
\hline & & 1.25 & 71 \\
\hline & & 1.5 & 97 \\
\hline & & 1.75 & 126 \\
\hline & & 2.0 & 155 \\
\hline \multirow[t]{10}{*}{ constant-power load } & $3-4$ & 1.0 & 39 \\
\hline & & 1.25 & 62 \\
\hline & & 1.5 & 84 \\
\hline & & 1.75 & 107 \\
\hline & & 2.0 & 128 \\
\hline & $3-5$ & 1.0 & 42 \\
\hline & & 1.25 & 68 \\
\hline & & 1.5 & 94 \\
\hline & & 1.75 & 123 \\
\hline & & 2.0 & 153 \\
\hline
\end{tabular}

current, the results indicate a range of possible fault distances - and vice versa.

\section{REFERENCES}

[1] H. Yuan, Z. Guan, H. Kong, B. Zhang, R. Huang, W. Wu, Y. Li, and D. Guo, "Impact of renewable energy integration on overcurrent protection in distribution network," in Control and Decision Conference (CCDC), 2015 27th Chinese, May 2015, pp. 5090-5095.

[2] V. Vega-García, J. C. Cebrian, and N. Kagan, "Evaluation of probability functions related to short circuit random variables using power quality meters," in 2010 IEEE/PES Transmission and Distribution Conference and Exposition: Latin America (T D-LA), 2010, pp. 712-718.

[3] S. Lotfifard, M. Kezunovic, and M. J. Mousavi, "A systematic approach for ranking distribution systems fault location algorithms and eliminating false estimates," IEEE Transactions on Power Delivery, vol. 28, no. 1, pp. 285-293, Jan 2013. 
TABLE II

RESULTS: POSSIBLE SHORT-CIRCUIT CURRENTS CALCULATED WITH A 200 [FT] FAULT DISTANCE IN FIG. 4

\begin{tabular}{|c|c|c|c|}
\hline Setting & Lateral & $P_{D G}(\mathrm{MW})$ & fault current $(\mathrm{kA})$ \\
\hline \multirow[t]{10}{*}{ constant-impedance load } & $3-4$ & 1.0 & 10.77 \\
\hline & & 1.25 & 10.92 \\
\hline & & 1.5 & 11.07 \\
\hline & & 1.75 & 11.22 \\
\hline & & 2.0 & 11.36 \\
\hline & $3-5$ & 1.0 & 10.86 \\
\hline & & 1.25 & 11.04 \\
\hline & & 1.5 & 11.23 \\
\hline & & 1.75 & 11.42 \\
\hline & & 2.0 & 11.62 \\
\hline \multirow{10}{*}{ constant-current load } & $3-4$ & 1.0 & 10.87 \\
\hline & & 1.25 & 11.02 \\
\hline & & 1.5 & 11.17 \\
\hline & & 1.75 & 11.33 \\
\hline & & 2.0 & 11.48 \\
\hline & $3-5$ & 1.0 & 10.96 \\
\hline & & 1.25 & 11.14 \\
\hline & & 1.5 & 11.33 \\
\hline & & 1.75 & 11.53 \\
\hline & & 2.0 & 11.74 \\
\hline \multirow{10}{*}{ constant-power load } & $3-4$ & 1.0 & 10.72 \\
\hline & & 1.25 & 10.87 \\
\hline & & 1.5 & 11.02 \\
\hline & & 1.75 & 11.17 \\
\hline & & 2.0 & 11.32 \\
\hline & $3-5$ & 1.0 & 10.81 \\
\hline & & 1.25 & 11.00 \\
\hline & & 1.5 & 11.20 \\
\hline & & 1.75 & 11.39 \\
\hline & & 2.0 & 11.59 \\
\hline
\end{tabular}

[4] M. Majidi, A. Arabali, and M. Etezadi-Amoli, "Fault location in distribution networks by compressive sensing," Power Delivery, IEEE Transactions on, vol. 30, no. 4, pp. 1761-1769, Aug 2015.

[5] L. Souto, G. Manassero, and S. G. Di Santo, "Fault location in distribution feeders with distributed generation," in 2016 Clemson University Power Systems Conference (PSC), March 2016, pp. 1-6.

[6] M. Dragomir, M. Istrate, and A. Dragomir, "Review of error sources in one-end data impedance-based fault location algorithms," in 2018 International Conference and Exposition on Electrical And Power Engineering (EPE), Oct 2018, pp. 0315-0320.

[7] E. Muljadi, N. Samaan, V. Gevorgian, Jun Li, and S. Pasupulati, "Short circuit current contribution for different wind turbine generator types," in IEEE PES General Meeting, 2010, pp. 1-8.

[8] R. Darbali-Zamora, J. Hernandez-Alvidrez, A. Summers, N. Gurule, M. Reno, and J. Johnson, "Distribution feeder fault comparison utilizing a real-time power hardware-in-the-loop approach for photovoltaic system applications," in 2019 IEEE 46th Photovoltaic Specialists Conference (PVSC), 2019, pp. 2916-2922.

[9] I. A. Essackjee and R. T. F. Ah King, "Fault level studies in a low voltage distribution network in the presence of small scale distributed generations- case study for mauritius," in 2019 International Conference on Advances in Big Data, Computing and Data Communication Systems (icABCD), 2019, pp. 1-5.

[10] P. P. Barker and R. W. De Mello, "Determining the impact of distributed generation on power systems. i. radial distribution systems," in 2000 Power Engineering Society Summer Meeting (Cat. No.00CH37134), vol. 3, July 2000 , pp. $1645-1656$ vol. 3 . 